(C) 1984. The Genetical Society of Great Britain

\title{
CHROMOSOMAL LOCALISATION OF FITNESS MODIFIERS OF DIAZINON RESISTANCE GENOTYPES OF LUCILIA CUPRINA
}

\author{
J. A. MCKENZIE AND ALISON PURVIS \\ Department of Genetics, University of Melbourne, Parkville, Victoria, 3052, Australia
}

Received 17.v.84

\section{SUMMARY}

\begin{abstract}
Previous results demonstrated that modification of the genetic background producea changes in the fitness of genotypes at the diazinon resistance locus in Lucilia cuprina (McKenzie et al., 1982). Fitness sets were estimated from population cage studies following disruption of the field genome by generations of backcrossing of a resistant field strain to males of a susceptible laboratory strain.

The present experiments report the results of a similar procedure where females of the laboratory strain have been used as the recurrent backcross parent. In this case recombination between the field and laboratory genomes is precluded during backcrossing. Population cage results were analogous to those of the previous experiments suggesting fitness modifiers were unlinked to the diazinon resistance locus on chromosome IV. The use of chromosome substitution lines provided confirmation. Major fitness modifiers were mapped to chromosome III.

The relevance of changes in the fitness sets of insecticide resistance genotypes, because of genetic background modification, to models of the evolution of insecticide resistance is discussed.
\end{abstract}

\section{INTRODUCTION}

The maintenance of stable agricultural ecosystems depends on the availability of effective pesticides. However, the capacity of insects to develop resistance is well documented (Brown and $\mathrm{Pal}, 1971$; Georghiou, 1980) and the ready availability of novel insecticides is limited because of the difficulties of development, registration and cross resistance (Forsyth and Lehman, 1979; Georghiou, 1980). Therefore, strategies should be devised to maximise the duration of efficacy of a chemical by minimising the probability of resistance developing. There is no shortage of models with this objective (see for example, Comins, 1977; 1979; Curtis, 1981; Curtis et al., 1978; Georghiou 1980; Georghiou and Taylor, 1977a; 1977b; Tabashnik and Croft, 1982; Taylor and Georghiou, 1979; Wood and Mani, 1981).

The models implicate many factors under broad genetical, biological and operational headings as being potentially important in determining the rate at which resistance evolves (Georghiou and Taylor, 1977a). It should be noted that the majority of studies are simulations and the relevance of these factors in natural populations has been less commonly assessed. Both theoretical and field studies generally explain the evolution of insecticide resistance of economic significance by allelic changes at a single locus (Brown and Pal, 1971; Whitten and McKenzie, 1982). It is usual for resistant genotypes to have a lower fitness than susceptible genotypes prior to the introduction of an insecticide. However, once resistance has become established continued selection by the insecticide may result in increased relative fitness of resistant genotypes in environments free of the insecticide (Bøggild and Keiding, 1958; Abedi and Brown, 1960; Keiding, 1967), although the 
genetics of these changes remains obscure. Such fitness modification has been predicted by the models (Georghiou, 1980) and documented for diazinon resistance in the Australian sheep blowfly, Lucilia cuprina (McKenzie et al., 1982). In the Lucilia system the possibility of defining the genetic basis of the change exists.

McKenzie et al., (1982) found that in the absence of diazinon the current field genetic background gave similar viability and fitness estimates for susceptible $(++)$, intermediate $(R+)$ and resistant $(R R)$ genotypes of the diazinon resistance locus on chromosome IV. Disruption of the field genome by repeated backcrossing to a laboratory strain suggested ++ genotypes became relatively fitter, in population cages, with increased backcrossing thus recreating the fitness set observed soon after the initial development of resistance (Arnold and Whitten, unpublished). As the backcrossing regime of McKenzie et al., (1982) used $\mathrm{R}+$ females with males of the ++ laboratory strain it was not possible to determine if the fitness modification was due to gene(s) linked with the resistance locus. Recombination is not usual in L. cuprina males (Foster et al., 1981) so an initial method of distinguishing between linked and unlinked modifier(s) is to use the reciprocal backcrossing procedure. Population cage results similar to those of the original experiments would argue strongly for modifiers that are not on the same chromosome as the resistance locus. However, if population cage trends are independent of the degree of backcrossing an hypothesis of linked modifiers would be favoured.

The experiments reported here support the former hypothesis and indicate, through the use of chromosome substitution lines, that the gene(s) having the major influence in modifying the fitness of diazinon resistance genotypes are located on chromosome III.

\section{Materials and Methods}

\section{(i) Strains}

Three strains were used in the experiments. (i) A standard reference susceptible strain, SWT, genotypically ++ at the diazinon resistance locus $\mathbf{R}_{1}$ located on chromosome IV (Foster et al,, 1981). (ii) A strain, $\mathbf{M}_{1} 5$ of equivalent resistance status to SWT but with a recessive marker for each autosome (II-black puparium ( $b p)$; III rusty $(r u)$; IV golden $(g l) ; \mathrm{V} \mathrm{M}_{1}$ veinless $\left(\mathrm{m}_{1}\right)$; VI yellow $\left.(y)\right)$ Whitten et al., 1975). (iii) A pure breeding resistance strain, $R R$, derived from flies collected in Victoria in 1981. The resistance phenotype of this strain is consistent with its being fixed for the $\mathbf{R}_{1 \mathrm{~A}}$ allele (McKenzie et al., 1980). The current backcross experiments began after RR had been in the laboratory for five generations. The experiments involving $M_{1} 5$ began approximately twenty generations after $R R$ was established.

\section{(ii) Population cages}

\section{(a) Establishment}

\section{Insertion of wild type genetic background into $R R$ genotype}

The SWT and RR strains were crossed and $F_{1}$ individuals used to establish the generation 0 of backcrossing population cage. $F_{1}$ males were 
also backcrossed to SWT virgin females and the R+ male progeny class ascertained by the topical application of $0.5 \mu 1$ of 0.01 per cent $(v / v)$ diazinon to the thorax of the fly using a Drummond micropipette (McKenzie et al., 1982). Surviving males of each generation were then backcrossed to SWT females, the procedure being repeated for ten generations of backcrossing. Population cages were established from $R+$ individuals after two, four, six, eight and ten generations of backcrossing.

\section{Generation of chromosome marker lines}

The $M_{1} 5$ and $R R$ strains were crossed and $F_{1}$ individuals used to establish a population cage.

$F_{1}$ males were also backcrossed to virgin $M_{1} 5$ females and flies of $\mathrm{gl}^{+}$ phenotype (and therefore $\mathrm{R}+$ ) segregating for one other chromosomal marker (e.g., $\left.b p / b p^{+} ; r u / r u ; g l / g l^{+} ; m_{1} / m_{1} ; y / y\right)$ chosen and used to establish population cages by crossing males and females of the same phenotype.

\section{(b) Maintenance}

Each population cage was started with forty flies of each sex and maintained under normal laboratory conditions (Whitten et al., 1975) in a discrete generation design. The cages were continued at each generation from a random sample of forty males and females. Three cages were run for each comparison.

\section{(c) Scoring of resistance status and marker frequency}

A random sample of twenty-five males and twenty-five females was chosen from each generation of each population cage and tested with $0.5 \mu \mathrm{l}$ of 0.01 per cent $(\mathrm{v} / \mathrm{v})$ diazinon to determine the proportion of ++ individuals in the population (McKenzie et al., 1982). In appropriate cages the frequency of visible markers was estimated from 100 randomly chosen flies and/or pupae (for $b p$ marker).

\section{Results}

\section{(a) Wild type background}

The percentage of ++ individuals observed in the population cages can be related to the genetic background of the original populations (fig. 1). For nought, two and four generations of backcrossing the populations essentially maintained the same percentage of ++ individuals across subsequent generations. However, in the six, eight and ten generations of backcrossing cages there was a consistent increase in ++ frequency with time, the rate being generally correlated with the level of backcrossing. Similar trends were observed in all trials (standard error range for related cages at a particular generation 0.00-8.49, angular scale).

The results for the current experiments in which $R+$ males were used in the backcross regime are comparable to those for previous experiments in which $\mathrm{R}+$ females were backcrossed to ++ (fig. 1). In each case, six or more generations of backcrossing resulted in an increase of the percentage of ++ individuals as population cage generation increased. 


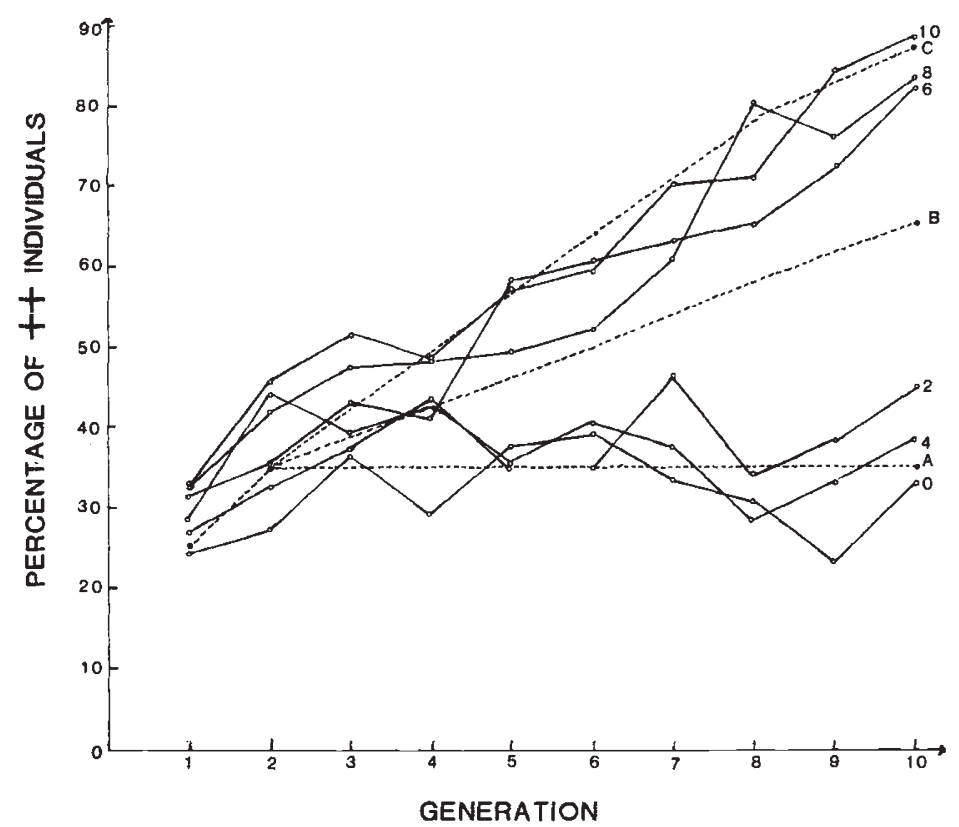

FIG. 1. Percentage of ++ individuals, averaged over three trials, at each generation in population cages established after $0,2,4,6,8$ and 10 generations of backcrossing $\mathrm{R}+$ males to a ++ strain. Lines of best fit (-- - ) for cages from previous experiments (McKenzie et al., 1982) where R+ females were backcrossed to the ++ strain for 0,3 (A), 6 (B) and 9 (C) generations are provided for comparison.

\section{(b) $M_{1} 5$ background}

The percentage of ++ (homozygous susceptible) individuals remained similar across generations of the population cages originally generated from $F_{1}$ progeny of the cross between the $M_{1} 5$ and $R R$ strains (fig. 2). There was again excellent agreement between trials (standard error range $0 \cdot 71-5 \cdot 15$, angular scale) and this also applied to the frequency of markers observed in each of the population cages. The frequency of each autosomal marker declined during the ten generations the cages were run (fig. 2). There was no association between the allelic frequencies at the resistance locus and those at the marker loci.

\section{(c) Chromosome marker population cages}

An increase in the percentage of ++ individuals was observed over generations in population cages in which chromosomes II, V and VI were derived from the RR strain (fig. 3). However, in cages in which chromosome III was derived from the RR strain the percentage of ++ individuals fluctuated around the initial value. Similar trends were observed for each trial (standard error range $0 \cdot 89-5 \cdot 30$, angular scale) reinforcing the difference of the trends in the chromosome III cages to those of the other chromosomes.

The percentage of particular chromosomal markers was also similar between trials (standard error range $0 \cdot 82-8 \cdot 57$, angular scale) however, in 

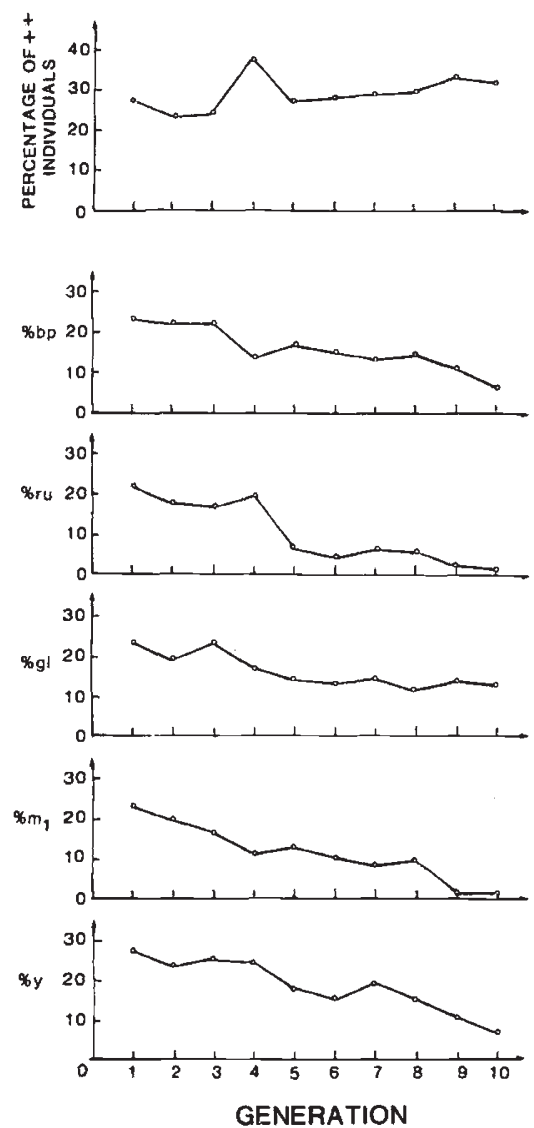

FIG. 2. Percentage of ++ individuals, averaged over three trials, at each generation in population cages started with the progeny of a cross between the $M_{1} 5$ and RR strains. The percentage of each autosomal marker at each generation is also given.

contrast to the $M_{1} 5$ background cages (fig. 2), the markers fluctuated about the starting frequency or, in the case of $b p$, increased with population cage generation (fig. 4). Irrespective of the other chromosomes segregating the frequency of the $g l$ marker showed similar trends in all cages.

\section{Discussion}

The population of ++ individuals in population cages with,$++ \mathbf{R}+$ and RR genotypes fluctuated about the initial frequency if the cages were started with $\mathrm{R}+$ individuals from a cross between a laboratory $(++)$ and a field (RR) strain (fig. 1). Similar results were gained after two and four generations of backcrossing to the laboratory strain. However, the proportion of ++ individuals increased over generations if the field genome was disrupted by six or more generations of backcrossing (fig. 1). These results confirm those previously reported (McKenzie et al., 1982) and demonstrate the importance of the genetic background to the fitness of genotypes at the resistance locus. Furthermore, the similarity of changes in ++ frequency 


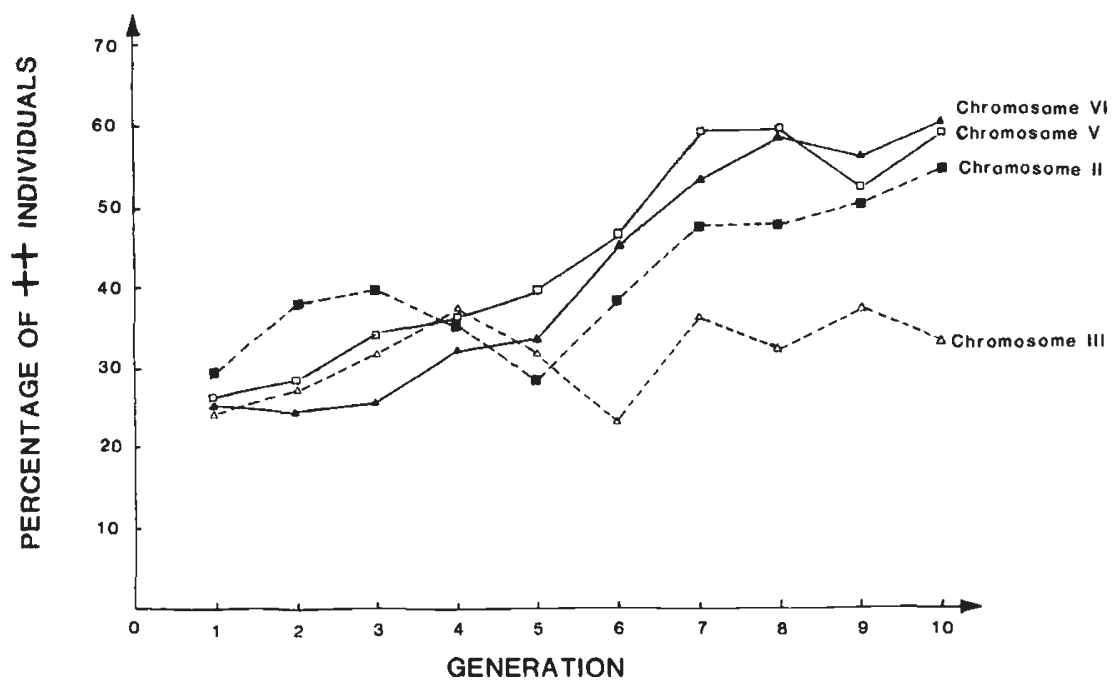

Fig. 3. Percentage of ++ individuals, averaged over three trials, at each generation for population cages segregating for $\mathrm{gl} / \mathrm{gl}^{+}$(chromosome IV) and one other chromosome $\left(b p / b p^{+} ; r u / r u ; g l / g l^{+} ; \mathrm{m}_{1} / \mathrm{m}_{1} ; y / y ;\right.$ chromosome II. $b p / b p ; r u / r u^{+} ; g l / g l^{+} ; m_{1} / m_{1}$; $y / y ;$ chromossome III. bp/bp; ru/ru; $\mathrm{gl} / \mathrm{gl}^{+} ; \mathrm{m}_{1} / \mathrm{m}_{1}^{+} ; y / y ;$ chromosome V. $b p / b p ; r u / r u$; $g l / g l^{+} ; \mathrm{m}_{1} / \mathrm{m}_{1} ; y / y^{+} ;$chromosome VI).
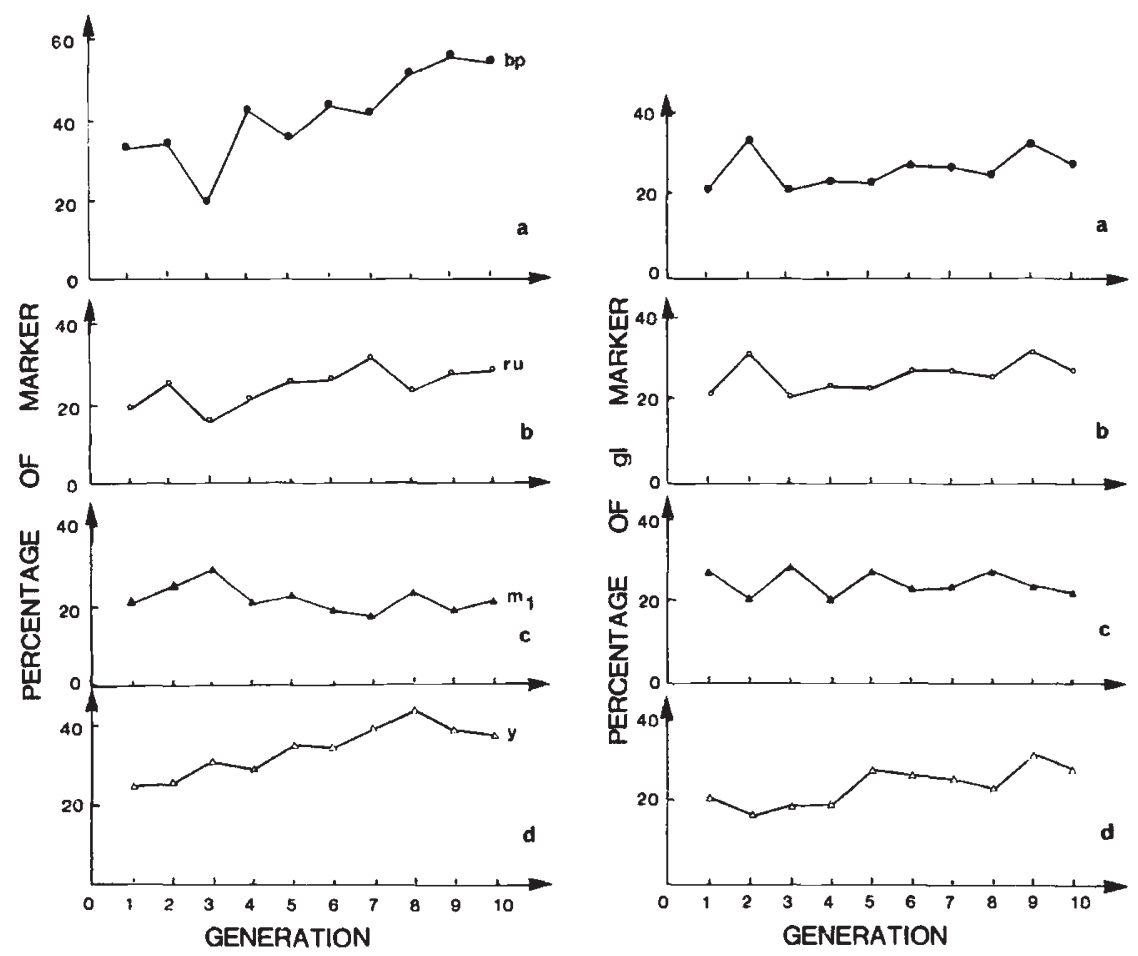

FIG. 4. The percentage, averaged over three trials, of each chromosomal marker and of the chromosome IV marker $(g l)$ in each of the chromosomal marker population cages. Chromosome II (a), chromosome III (b), chromosome V (c), chromosome VI (d). 
in population cages started after similar numbers of generations of backcrossing in both sets of experiments argues that any major modifiers are not on the same chromosome as the resistance locus since the present backcross regime precluded recombination between field and laboratory genomes during the backcrossing procedure. Such recombination had been allowed in the prevous experiments (McKenzie et al., 1982).

Given these results it may appear surprising that so many generations of backcrossing are required to disrupt the field genome to the extent necessary to alter the original fitness set. After three generations of recurrent backcrossing to SWT only 6.25 per cent of the field genome remains in $\mathrm{R}+$ individuals used to initiate the population cages. Therefore, on this basis the results of McKenzie et al., (1982) appeared to support the possibility of the modifier(s) being linked to the resistance locus. The present results do not support this and indicate that the population cage method of fitness assessment is screening only for modifiers that have a significant effect on the fitness sets associated with resistance genotypes. In natural populations the selection process will be at least as rigorous and may also select for minor modificational change that is not identified in the laboratory experiments.

More precise localisation of the fitness modifiers required the use of a chromosomal marker stock. Initially, it was necessary to demonstrate that the modifiers acted in this genetic background. It was observed that for cages initiated with the progeny of a $M_{1} 5$ by $R R$ cross the ++ frequency remain constant over cage generations (fig. 2). Therefore, the modifiers were effective in this background as the result paralleled those of cages started from the RR x SWT $(++)$ cross (fig. 1).

Chromosome substitution lines established from the chromosome marker stock allowed comparison of ++ frequency in population cages segregating for a single field autosome (in conjunction with chromosome IV which has the resistance locus). If a major modifier is present on a particular chromosome the frequency of ++ was expected to remain constant in cages segregating for that chromosome as the presence of the modifier would establish a fitness set where,$++ R+$ and $R R$ genotypes have similar fitness. That is, the fitness currently observed in the field background. The fitness set defined by the absence of the modifier predicts an increase in ++ frequency with cage generation. On this basis major modifier activity appeared to be associated with chromosome III (fig. 3).

The rate of change in ++ frequency in the chromosome substitution line population cages other than for chromosome III is less rapid than that observed for six or more generations of backcrossing (figs 1 and 3 ). This most probably reflects general differences in the stocks being used in each experiment but raises the possibility of some fitness modifier activity associated with chromosome IV, which is segregating in all population cages, or of modifiers of minor influence on autosomes other than chromosome III. However, the effect of chromosome III remains clear; the relative importance of this chromosome to fitness modification is unambiguous.

The changes in gene frequency of the chromosomal markers in the population cages are of interest. When all of the autosomes were segregating for both marker and field chromosomes the markers were selected against (fig. 2). If only chromosome IV and one other chromosome were segregating this selective disadvantage was not apparent (fig. 4). The fitness of a marker 
therefore also depends on the genetic background in which the comparison is made (King and Dawson, 1972). However, in all comparisons, there was independence between the frequency of a marker and the proportion of ++ individuals in a cage. Changes in gene frequency at the resistance locus cannot be ascribed to effects associated with a particular marker.

The present results emphasise that the fitness set at a locus may be infiuenced by genetic background (Merrell, 1965; Dawson, 1970; Wills and Nichols, 1972; Jones and Yamazaki, 1974). In this instance, the rate of evolution of fitness modification of the diazinon locus (McKenzie et al, 1982) and the localization of modifier activity to a single chromosome suggest few genes may be involved. This affords the opportunity for precise mapping which may assist in allowing the definition of the functional relationship between the modifier(s) and the resistance locus but it should be noted that fitness modification does not alter the resistance phenotypic status of,$++ R+$ and RR genotypes. Dosage mortality curves for each genotype have remained constant over a considerable period (Arnold and Whitten, 1976; McKenzie et al., 1980). While laboratory selection programmes can select background genetic effects that change the phenotypic resistance status, this variation is apparently rarely selected in the field (McKenzie et al., 1980). Conversely, there has been natural selection of a genetic background that modifies the fitness of resistance genotypes. Hence, it is essential to note that there may not be a simple relationship between insecticide resistance status and the fitness of genotypes defining that status. (Whitten and McKenzie, 1982; Roush and Plapp, 1982; McKenzie, 1983).

Each of these conclusions adds to the information on evolution of insecticide resistance in $L$. cuprina and challenges the adequacy of models where fitness sets are based only on the presence or the absence of an insecticide (McKenzie-and Whitten, 1982; 1984; Whitten and McKenzie, 1982). General models have the capacity to focus on areas of potential relevance (Georghious, 1980). However, if the most effective management practices are to be identified information must be accumulated for specific systems. This is not to decry the value of the general model. Rather, it is to ask whether the conclusions of the model require modification after finer scale analysis. As $L$. cuprina can be manipulated in both the field and laboratory (Foster et al., 1975; Foster, 1979; Whitten et al., 1980), has a well mapped genome (Whitten et al., 1975) and is currently experiencing a change in the major chemical control agent (diazinon to vetrazin) this system provides a rare chance to adequately address this question.

Acknowledgments. Drs Max Whitten and Phil Batterham are thanked for discussion and comments on the manuscript. Nan Austin, Jenny Fegent, Julie Lycette and Tracey Collings provided excellent technical assistance at various stages of the work. The project was supported by the Australian Research Grants Scheme and the Australian Wool Corporation Research Trust Fund.

\section{REFERENCES}

ABEDI, Z. H. AND BROWN, A. W. A. 1960. Development and reversion of DDT-resistance in Aedes aegypti. Can. J. Genet. Cytol., 2, 252-261.

ARNOLD, J. T. A. AND WHITTEN, M. J. 1976. The genetic basis for organophosphorus resistance in the Australian sheep blowfly, Lucilia cuprina (Wiedemann) (Diptera, Calliphoridae). Bull. Entomol. Res., 66, 561-568. 
BøGGILD, I. AND KEIDING, J. 1958. Competition in housefly larvae. Experiments involving a DDT-resistant and a susceptible strain. Oikos, 9, 1-25.

BROWN, A. W. A. AND PAL, R. 1971. Insecticide Resistance in Arthropods. W.H.O. Geneva.

COMINS, H. N. 1977. The development of insecticide resistance in the presence of migration. J. Theor. Biol, 64, 177-197.

COMINS, H. N. 1979. The management of pesticide resistance: models. In Genetics in Relation to Insect Management. M. A. Hoy and J. J. McKelvey Jr. (ed.). Rockefeller Foundation U.S.A., pp. 55-69.

CURTIS, C. F. 1981. Possible methods of inhibiting or reversing the evolution of insecticide resistance in mosquitoes. Pestic. Sci., 12, 557-564.

CURTIS, C. F., COOK, L. M. AND WOOD, R. J. 1978. Selection for and against insecticide resistance and possible methods of inhibiting the evolution of resistance in mosquitoes. Ecol. Entomol., 3, 273-287.

DAWSON, P. S. 1970. Linkage and the elimination of deleterious mutant genes from experimental populations. Genetica, 41, 147-169.

FORSYTH, B. A. AND LEHMAN, P. G. 1979. Insecticides for the future-likelihood of new compounds. In National Symposium on Sheep Blowfly and Flystrike in Sheep. Department of Agriculture, N.S.W., pp. 97-111.

FOSTER, G. G. 1979. Genetic control of Lucilia cuprina and the logistics of the CSIRO control programme. In National Symposium on the Sheep Blowfly and Flystrike in Sheep. Department of Agriculture, N.S.W., pp. 59-67.

FOSTER, G. G., KITCHING, R. L., VOGT, W. G. AND WHITTEN, M. J. 1975. Sheep Blowfly and its control in the pastoral ecosystem of Australia. Proc. Ecol. Soc. Aust., 9, 213-229.

FOSTER, G. G., WHITTEN, M. J., KONOVALOV, C., ARNOLD, J. T. A. AND MAFFI, G. 1981. Autosomal genetic maps of the Australian sheep blowfly, Lucilia cuprina dorsalis R-D. (Diptera: Calliphoridae), and possible correlations with the linkage maps of Musca domestica L. and Drosophila melanogaster (Mg.). Genet. Res., 37, 55-69.

GEORGHIOU, G. P. 1980. Insecticide resistance and prospects for its management. Residue Reviews, 76, 131-145.

GEORGHIOU, G. P. AND TAYLOR, C. E. 1977a. Genetic and biological influences in the evolution of insecticide resistance. J. Econ. Entomol., 70, 319-323.

GEORGHIOU, G. P. AND TAYLOR, C. E. 1977b. Operational influences in the evolution of insecticide resistance. J. Econ. Entomol, 70, 653-658.

JONES, J. S. AND YAMAZAKI, T. 1974. Genetic background and the fitness of allozymes. Genetics, $78,1185-1189$.

KEIDING, J. 1967. Persistence of resistant populations after relaxation of the selection pressure. World. Rev. Pest Control, 6, 115-130.

KING, C. E. AND DAWSON, P. S. 1972. Population biology and the Tribolium model. In Th. Dobzhansky, M. K. Hecht, and W. C. Steere (ed.). Evolutionary Biology, Vol. 5, AppletonCentury-Crofts, New York, pp. 133-227.

MCKENZIE, J. A. 1983. The evolution of insecticide resistance; consequences for control programmes. In 2nd National Symposium on Sheep Blowfly and Flystrike in Sheep. Department of Agriculture, N.S.W., pp. 222-231.

MCKENZIE, J. A., DEARN, J. M. AND WHITTEN, M. J. 1980. Genetic basis of resistance to diazinon in Victorian populations of the Australian sheep blowfly, Lucilia cuprina. Aust. J. Biol. Sci., 33, 85-95.

MCKENZIE, J. A. AND WHITTEN, M. J. 1982. Selection for insecticide resistance in the Australian sheep blowfly, Lucilia cuprina. Experientia, 38, 84-85.

MCKENZIE, J. A. AND WHITTEN, M. J. 1984. Estimation of the relative viabilities of insecticide resistance genotypes of the Australian sheep blowfly, Lucilia cuprina. Aust. J. Biol. Sci., $37,45-52$.

MCKENZIE, J. A., WHITTEN, M. J. AND ADENA, M. A. 1982. The effect of genetic background on the fitness of diazinon resistance genotypes of the Australian sheep blowfly, Lucilia cuprina. Heredity, 49, 1-9.

MERRELL, D. J. 1965. Competition involving dominant mutants in experimental populations of Drosophila melanogaster. Genetics, 52, 165-189.

ROUSH, R. T. AND PLAPP, F. W. Jr. 1982. Effects of insecticide resistance on biotic potential of the house fly (Diptera: Muscidae) J. Econ. Entomol., 75, 708-713.

TABASHNIK, B. E. AND CROFT, B. A. 1982. Managing pesticide resistance in crop-arthropod complexes: interactions between biological and operational factors. Environ. Entomol., $11,1137-1144$.

TAYLOR, C. E. AND GEORGHIOU, G. P. 1979. Suppression of insecticide resistance by aiteration of gene dominance and migration. J. Econ. Entomol., 72, 105-109. 
WHITTEN, M. J., DEARN, J. M. AND MCKENZIE, J. A. 1980. Field studies on insecticide resistance in the Australian sheep blowfly, Lucilia cuprina. Aust. J. Biol. Sci., 33, 725-735.

WHITTEN, M. J., FOSTER, G. G., ARNOLD, J. T. AND KONOVAlOV, C. 1975. The Australian sheep blowfly, Lucilia cuprina. In R. C. King, Vol. 3, (ed.). Handbook of Genetics, pp. 401-418, Plenum, New York.

WHITTEN, M. J. AND MCKENZIE, J. A. 1982. The genetic basis for pesticide resistance. In K. E. Lee (ed). Proc. 3rd Aust. Conf. Grassland Invertebrate Ecology. S.A. Govt. Printer, Adelaide, pp. 1-16.

WILLS, C. AND NICHOLS, L. 1972. How genetic background masks single-gene heterosis in Drosophila. Proc. Nat. Acad. Sci. U.S.A., 69, 323-325.

WOOD, R. J. AND MANI, G. S. 1981. The effective dominance of resistance genes in relation to the evolution of resistance. Pestic. Sci., 12, 573-581. 